\title{
Erratum to: Signal detection theory and vestibular thresholds: I. Basic theory and practical considerations
}

\author{
Daniel M. Merfeld
}

Published online: 9 March 2012

(c) Springer-Verlag 2012

Erratum to: Exp Brain Res (2011) 210:389-405

DOI 10.1007/s00221-011-2557-7

In the original publication of this article, several figures were altered during the typesetting process. These alterations resulted in a substantive error in panel $\mathrm{h}$ of Fig. 1. In addition, two figure legends included typos. These errors are fixed by reprinting corrected Figs. 1 and 2 and figure legends.

The online version of the original article can be found under doi:10.1007/s00221-011-2557-7.

D. M. Merfeld ( $\square)$

Jenks Vestibular Research Laboratory,

Massachusetts Eye and Ear Infirmary,

Boston, MA, USA

e-mail: dan_merfeld@meei.harvard.edu 

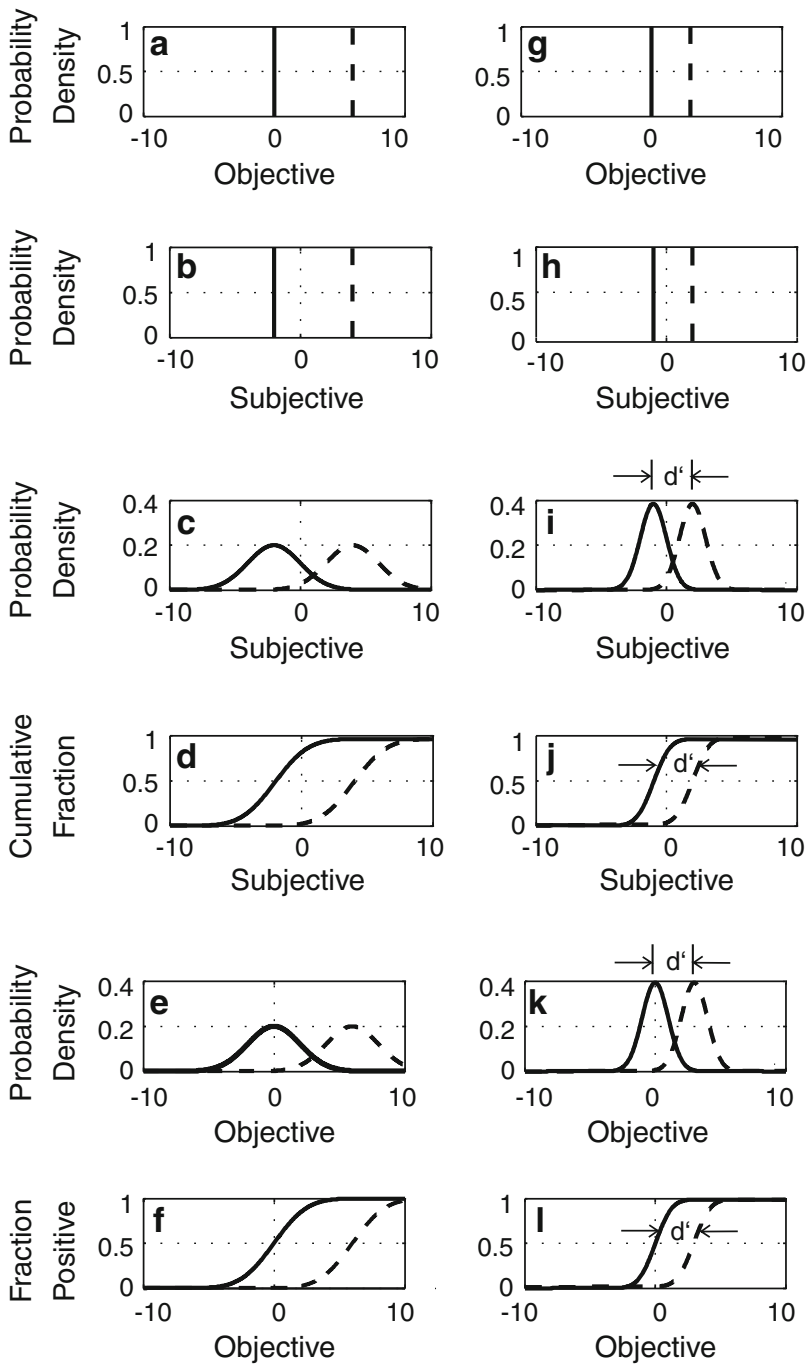

Fig. 1 a Objective stimuli having amplitudes of 0 (the null stimulus) and 6. b A vestibular bias of -2 yields the sensed stimuli of -2 and +4 . c A probability density function (PDF) represents that the perceived amplitude will have some variation $(\sigma=2)$ due to noise. d A cumulative distribution function (CDF) is calculated as the integral of the PDF. e, $\mathbf{f}$ The PDF and CDF in objective coordinates. The right column (g through $\mathbf{l}$ ) represents the same quantities but normalized by 2 -the standard deviation from the left column. In these normalized units, $\mathbf{g}$ the objective stimuli are 0 and $3, \mathbf{h}$ the vestibular bias is -1 yielding sensed stimuli of -1 and +2 , (i through l) the noise has a standard deviation of 1 . To represent vestibular bias (i.e., shifts in the PSE), subjective coordinates are shifted with respect to the objective coordinates; note that objective and subjective curves remain aligned on the page
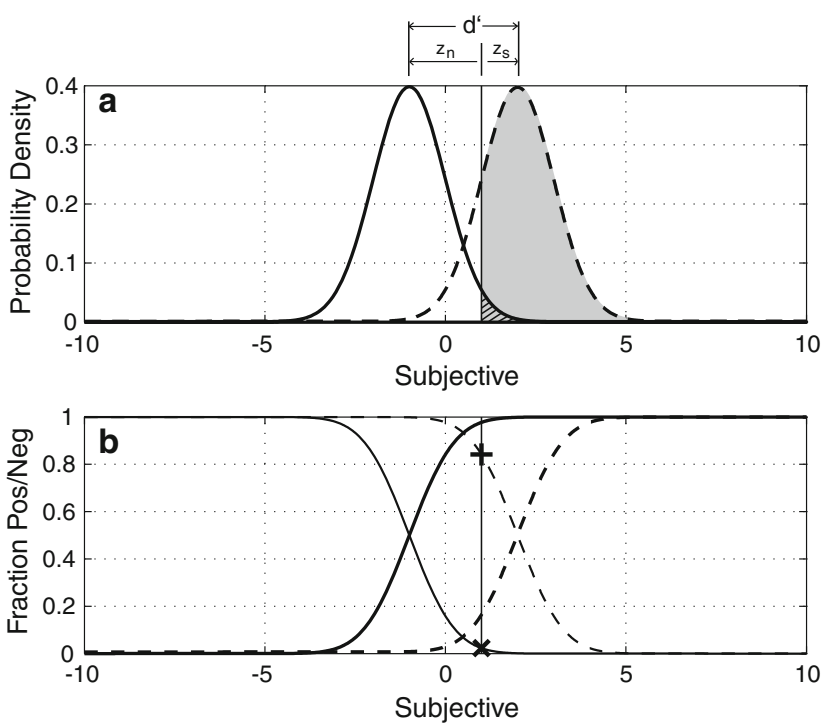

Fig. 2 a Subjective PDFs for sensed stimuli having mean amplitudes of -1 and +2 . The shaded area represents the hit rate. The hatched area represents the false alarm rate. The thick curves in b show the CDFs for these distributions. The thin curves show one minus these CDFs. The + represents the hit rate. The $x$ represents the false alarm rate 